\title{
TWO WEIGHTED INEQUALITIES FOR MAXIMAL FUNCTIONS RELATED TO CESÀRO CONVERGENCE
}

\section{A. L. BERNARDIS and F. J. MARTÍN-REYES}

(Received 4 April 2000; revised 23 November 2001)

\author{
Communicated by A. H. Dooley
}

\begin{abstract}
We characterize the pairs of weights $(u, v)$ for which the maximal operator

$$
M_{\alpha}^{-} f(x)=\sup _{R>0} R^{-1-\alpha} \int_{x-2 R}^{x-R}|f(s)|(x-R-s)^{\alpha} d s, \quad-1<\alpha<0,
$$

is of weak and restricted weak type $(p, p)$ with respect to $u(x) d x$ and $v(x) d x$. As a consequence we obtain analogous results for

$$
M_{\alpha} f(x)=\sup _{R>0} R^{-1-\alpha} \int_{R<|x-y|<2 R}|f(y)|(|x-y|-R)^{\alpha} d y .
$$

We apply the results to the study of the Cesàro- $\alpha$ convergence of singular integrals.

2000 Mathematics subject classification: primary 42B25; secondary: 42B20, 42B08.

Keywords and phrases: Cesàro maximal operator, Cesàro- $\alpha$ convergence, weights, singular integrals.
\end{abstract}

\section{Introduction}

Let $M_{\alpha}$ be the maximal operator defined at a measurable function $f$ on the real line by

$$
M_{\alpha} f(x)=\sup _{R>0} \frac{1}{R^{1+\alpha}} \int_{R<|x-y|<2 R}|f(y)|(|x-y|-R)^{\alpha} d y, \quad-1<\alpha<0 .
$$

This operator occurs in a natural way when one studies the Cesàro- $\alpha$ convergence of singular integrals [2]. Alternatively,

$$
M_{\alpha} f(x)=\sup _{R>0}|f| * \varphi_{R}(x),
$$

The first author was supported by CONICET, Prog. CAI+D - UNL and PICT 98 (Código 03-04186). The second author was partially supported by D.G.E.S. grant (BFM2001-1638) and Junta de Andalucía. (C) 2003 Australian Mathematical Society 1446-7887/03 \$A2.00+0.00 
where $\varphi_{R}(x)=R^{-1} \varphi\left(R^{-1} x\right)$ and $\varphi(s)=(|s|-1)^{\alpha} \chi_{(1,2)}(|s|)$. From this point of view, $M_{\alpha}$ is a particular case of the operator studied in [5]. It follows from [5, Theorem 1] that $M_{\alpha}$ is of restricted weak type $(1 /(1+\alpha), 1 /(1+\alpha))$ and that it is not of weak type $(1 /(1+\alpha), 1 /(1+\alpha))$ with respect to the Lebesgue measure when $\alpha<0$ (notice that $M_{0}$ is equivalent to the Hardy-Littlewood maximal operator).

Weighted inequalities for $M_{\alpha}$ were studied in [2] and [3]. In [3] we obtained a characterization of weighted inequalities for a single weight. The doubling condition plays an essential role in the proof of this characterization; it was also the key reason why we were not able to study the two-weight case in [3].

In this paper we develop a different approach to the study of weighted inequalities for $M_{\alpha}$ which enables us to obtain a characterization of the two-weighted weak and restricted weak type inequalities for $M_{\alpha}$. This new method consists of the study of one-sided versions of $M_{\alpha}$

and

$$
M_{\alpha}^{-} f(x)=\sup _{R>0} \frac{1}{R^{1+\alpha}} \int_{x-2 R}^{x-R}|f(y)|(x-R-y)^{\alpha} d y
$$

$$
M_{\alpha}^{+} f(x)=\sup _{R>0} \frac{1}{R^{1+\alpha}} \int_{x+R}^{x+2 R}|f(y)|(y-x-R)^{\alpha} d y .
$$

These operators are of interest because they naturally appear in the investigation of the Cesàro- $\alpha$ convergence of singular integrals with kernels supported in $(0, \infty)$ and in $(-\infty, 0)$.

The paper is organized as follows. In Section 2 we state and prove a characterization of two-weighted weak and restricted weak type inequalities for $M_{\alpha}^{-}, M_{\alpha}^{+}$and $M_{\alpha}$; in Section 3 we apply these results to the study of the existence of the singular integrals in the Cesàro- $\alpha$ sense.

Throughout the paper, $u, v$ and $w$ are weights, that is, positive measurable functions, $u(A)$ denotes the integral $\int_{A} u(s) d s, p^{\prime}$ denotes the conjugate exponent of $p, 1<$ $p<\infty$, and the letter $C$ means a positive constant that may change from one line to another.

\section{Two-weighted inequalities}

We start with the results for $M_{\alpha}^{-}$(analogous results hold for $M_{\alpha}^{+}$).

THEOREM 2.1. Let $u$ and $v$ be weights on $\mathbb{R}$ and let $-1<\alpha<0$. If $1<p<\infty$, then the following are equivalent:

(i) $M_{\alpha}^{-}$is of weak type $(p, p)$ with respect to $u(x) d x$ and $v(x) d x$, that is, there exists $C$ such that $u\left(\left\{M_{\alpha}^{-} f>\lambda\right\}\right) \leq C \lambda^{-p} \int|f|^{p} v$, for all $\lambda>0$ and all $f \in L^{p}(v)$. 
(ii) $(u, v)$ satisfies $A_{\bar{p}, \alpha}^{-}$, that is, there exists $C$ such that for any three numbers $a<b<c$,

$$
\left(\int_{b}^{c} u(s) d s\right)^{1 / p}\left(\int_{a}^{b} v^{1-p^{\prime}}(s)(b-s)^{\alpha p^{\prime}} d s\right)^{1 / p^{\prime}} \leq C(c-a)^{1+\alpha} .
$$

REMARK. Observe that if $\alpha<0$ and ess $\inf _{x \in(a, b)} v^{1-p^{\prime}}(x)>0$ for some interval $(a, b)$ then the two-weighted weak type $(p, p)$ inequality is not possible for $1<p \leq 1 /(1+\alpha)$ since (ii) does not hold in this case. However the operator $M_{\alpha}^{-}$is of restricted weak type $(1 /(1+\alpha), 1 /(1+\alpha))$ with respect to the Lebesgue measure. Therefore it is interesting to study the restricted weak type inequalities for pairs of weights.

THEOREM 2.2. Let $u$ and $v$ be weights on $\mathbb{R}$ and let $-1<\alpha<0$. If $1 \leq p<\infty$, then the following are equivalent:

(i) $M_{\alpha}^{-}$is of restricted weak type $(p, p)$ with respect to $u(x) d x$ and $v(x) d x$, that is, there exists $C$ such that $u\left(\left\{x: M_{\alpha}^{-} \chi_{E}(x)>\lambda\right\}\right) \leq C \lambda^{-p} v(E)$ for all $\lambda>0$ and all measurable $E \subset \mathbb{R}$.

(ii) $(u, v)$ satisfies $R A_{p, \alpha}^{-}$, that is, there exists $C$ such that for any three numbers $a<b<c$ and all measurable $E \subset \mathbb{R}$

$$
\left(\int_{b}^{c} u(s) d s\right)\left(\int_{a}^{b} \chi_{E}(s)(b-s)^{\alpha} d s\right)^{p} \leq C(c-a)^{(1+\alpha) p} \int_{a}^{b} \chi_{E}(s) v(s) d s .
$$

The corresponding results for $M_{\alpha}$ are obtained immediately from Theorem 2.1 and Theorem 2.2 and from the analogous ones for $M_{\alpha}^{+}$.

Now we shall state the results for $M_{\alpha}$ which generalize the weak and restricted weak type inequalities from [3] to the two-weight case.

THEOREM 2.3. Let $u$ and $v$ be weights on $\mathbb{R}$ and let $-1<\alpha<0$. If $1<p<\infty$, then the following are equivalent:

(i) $M_{\alpha}$ is of weak type $(p, p)$ with respect to $u(x) d x$ and $v(x) d x$.

(ii) $(u, v)$ satisfies $A_{p, \alpha}$, that is, there exists $C$ such that for any interval $I$

$$
\left(\int_{I} u(s) d s\right)^{1 / p}\left(\int_{2 I \backslash I} v^{1-p^{\prime}}(s) d(s, I)^{\alpha p^{\prime}} d s\right)^{1 / p^{\prime}} \leq C|I|^{1+\alpha},
$$

where $2 I$ is the interval with the same center and double length as $I$ and $d(s, I)$ is the Euclidean distance from $s$ to $I$.

THEOREM 2.4. Let $u$ and $v$ be weights on $\mathbb{R}$ and let $-1<\alpha<0$. If $1 \leq p<\infty$, then the following are equivalent: 
(i) $M_{\alpha}$ is of restricted weak type $(p, p)$ with respect to $u(x) d x$ and $v(x) d x$.

(ii) $(u, v)$ satisfies $R A_{p, \alpha}$, that is, there exists $C$ such that for every interval $I$ and all measurable $E \subset \mathbb{R}$

$$
\left(\int_{I} u(s) d s\right)\left(\int_{2 I \backslash I} \chi_{E}(s) d(s, I)^{\alpha} d s\right)^{p} \leq C|I|^{(1+\alpha) p} \int_{2 I \backslash I} \chi_{E}(s) v(s) d s
$$

The proofs of Theorem 2.3 and Theorem 2.4 are omitted since they are immediate corollaries of the previous results.

In order to prove Theorem 2.1 and Theorem 2.2 we use a noncentred maximal operator which is pointwise equivalent to $M_{\alpha}^{-}$. In what follows we define this operator and state the pointwise equivalence.

DEFINITION 2.5. For each $x \in \mathbb{R}$, let us consider the family of intervals $\mathscr{A}_{x}=$ $\{(a, b): b<x$ and $b-a \geq x-b\}$. We define the noncentred maximal operator $N_{\alpha}^{-}$ associated with $M_{\alpha}^{-}$as

$$
N_{\alpha}^{-} f(x)=\sup _{(a, b) \in \alpha_{x}} \frac{1}{(b-a)^{1+\alpha}} \int_{a}^{b}|f(s)|(b-s)^{\alpha} d s
$$

PROPOSITION 2.6. Let $-1<\alpha<0$. There exists a constant $C$ depending only on $\alpha$ such that $M_{\alpha}^{-} f \leq N_{\alpha}^{-} f \leq C M_{\alpha}^{-} f$, for all measurable functions $f$.

ProOF. The first inequality is obvious. Let $(a, b) \in \mathscr{A}_{x}, R=x-a$ and let $N$ be the natural number such that $x-2^{-N} R \leq b<x-2^{-N-1} R$. Then

$$
\begin{aligned}
\int_{a}^{b}|f(s)|(b-s)^{\alpha} d s \\
=\sum_{i=0}^{N-1} \int_{x-R / 2^{i}}^{x-R / 2^{i+1}}|f(s)|\left(x-\frac{R}{2^{i+1}}-s\right)^{\alpha}\left(\frac{b-s}{x-\left(R / 2^{i+1}\right)-s}\right)^{\alpha} d s \\
\quad+\int_{x-R / 2^{N}}^{b}|f(s)|(b-s)^{\alpha} d s=\mathrm{I}+\mathrm{II} .
\end{aligned}
$$

Since $(a, b) \in \mathscr{A}_{x}$,

$$
\mathrm{II} \leq \int_{x-2(x-b)}^{b}|f(s)|(b-s)^{\alpha} d s \leq(x-b)^{1+\alpha} M_{\alpha}^{-} f(x) \leq(b-a)^{1+\alpha} M_{\alpha}^{-} f(x) .
$$

On the other hand, since the function $s \rightarrow\left[(b-s) /\left(x-2^{-i-1} R-s\right)\right]^{\alpha}$ is decreasing 
on $\left(x-2^{-i} R, x-2^{-i-1} R\right), 0 \leq i \leq N-1$,

$$
\begin{aligned}
\mathrm{I} & \leq\left(\sum_{i=0}^{N-1}\left(b-\left(x-\frac{R}{2^{i}}\right)\right)^{\alpha} \frac{R}{2^{i+1}}\right) M_{\alpha}^{-} f(x) \\
& \leq M_{\alpha}^{-} f(x) \sum_{i=0}^{N-1} \int_{x-R / 2^{i}}^{x-R / 2^{i+1}}(b-s)^{\alpha} d s \leq C(b-a)^{1+\alpha} M_{\alpha}^{-} f(x),
\end{aligned}
$$

and we are done.

Proof of THEOREM 2.1. By Proposition 2.6, (i) is equivalent to the weighted weak type $(p, p)$ inequality for $N_{\alpha}^{-}$. Let $a<b<c$ and let $\bar{a}<a$ be such that $b-\bar{a}=c-a$. If we consider the function $f(s)=v^{1-p^{\prime}}(s)(b-s)^{\alpha\left(p^{\prime}-1\right)} \chi_{(a, b)}(s)$, then for all $x \in(b, c)$

$$
N_{\alpha}^{-} f(x) \geq \frac{1}{(b-\bar{a})^{1+\alpha}} \int_{a}^{b} v^{1-p^{\prime}}(s)(b-s)^{\alpha p^{\prime}} d s \equiv \lambda .
$$

This means that $(b, c) \subset\left\{N_{\alpha}^{-} f \geq \lambda\right\}$. Then (ii) follows from (i) (with $N_{\alpha}^{-}$) by a standard argument.

The implication (ii) implies (i) follows from the following proposition and the fact that the maximal operator $M_{u}^{-} g(x)=\sup _{h<x}\left(\int_{h}^{x}|g| u / \int_{h}^{x} u\right)$ is of weak type $(1,1)$ with respect to the measure $u(x) d x$.

PROPOSITION 2.7. Let $-1<\alpha<0$ and $p>1$. If $(u, v)$ satisfies $A_{p, \alpha}^{-}$, then there exists $C>0$ such that for every measurable function $f$

$$
N_{\alpha}^{-} f \leq C\left[M_{u}^{-}\left(|f|^{p} v u^{-1}\right)\right]^{1 / p} .
$$

PROOF. Let $x \in \mathbb{R}$ and $(a, b) \in \mathscr{A}_{x}$. First, let us assume that $4 \int_{b}^{x} u>\int_{a}^{x} u$. Since the pair $(u, v)$ satisfies $A_{p, \alpha}^{-}$, we have

$$
\begin{aligned}
\int_{a}^{b}|f(s)|(b-s)^{\alpha} d s & \leq\left(\int_{a}^{b}|f|^{p}(s) v(s) d s\right)^{1 / p}\left(\int_{a}^{b} v^{-p^{\prime} / p}(s)(b-s)^{\alpha p^{\prime}} d s\right)^{1 / p^{\prime}} \\
& \leq C\left(\int_{a}^{x}|f|^{p}(s) v(s) d s\right)^{1 / p}\left(\int_{b}^{x} u(s) d s\right)^{-1 / p}(x-a)^{1+\alpha} \\
& \leq C\left[M_{u}^{-}\left(|f|^{p} v u^{-1}\right)\right]^{1 / p}(x)(b-a)^{1+\alpha}
\end{aligned}
$$

Assume now that $4 \int_{b}^{x} u \leq \int_{a}^{x} u$. Let $\left\{x_{i}\right\}$ be the increasing sequence in $[a, x]$ defined by $x_{0}=a$ and $\int_{x_{i+1}}^{x} u=\int_{x_{i}}^{x_{i+1}} u=\frac{1}{2} \int_{x_{i}}^{x} u$. Let $N$ be such that $x_{N} \leq b<x_{N+1}$ (observe 
that $N \geq 2$ ). Then we have

$$
\begin{aligned}
\int_{a .}^{b}|f(s)|(b-s)^{\alpha} d s & =\sum_{i=0}^{N-2} \int_{x_{i}}^{x_{i+1}}|f(s)|(b-s)^{\alpha} d s+\int_{x_{N-1}}^{b}|f(s)|(b-s)^{\alpha} d s \\
& =\mathrm{I}+\mathrm{II} .
\end{aligned}
$$

By the $A_{p, \alpha}^{-}$condition and the fact that $\int_{x_{N-1}}^{x} u \leq 4 \int_{b}^{x} u$, we have

$$
\begin{aligned}
\mathrm{II} & \leq\left(\int_{x_{N-1}}^{b}|f|^{p}(s) v(s) d s\right)^{1 / p}\left(\int_{x_{N-1}}^{b} v^{-p^{\prime} / p}(s)(b-s)^{\alpha p^{\prime}} d s\right)^{1 / p^{\prime}} \\
& \leq C\left[M_{u}^{-}\left(|f|^{p} v u^{-1}\right)\right]^{1 / p}(x)(b-a)^{1+\alpha}
\end{aligned}
$$

On the other hand, since the function $s \rightarrow\left[(b-s) /\left(x_{i+1}-s\right)\right]^{\alpha}$ is decreasing in the interval $\left(x_{i}, x_{i+1}\right), 0 \leq i \leq N-2$, we obtain

$$
\begin{aligned}
& \int_{x_{i}}^{x_{i+1}}|f(s)|(b-s)^{\alpha} d s \leq\left(\frac{b-x_{i}}{x_{i+1}-x_{i}}\right)^{\alpha} \int_{x_{i}}^{x_{i+1}}|f(s)|\left(x_{i+1}-s\right)^{\alpha} d s \\
& \quad \leq\left(\frac{b-x_{i}}{x_{i+1}-x_{i}}\right)^{\alpha}\left(\int_{x_{i}}^{x_{i+1}}|f|^{p}(s) v(s) d s\right)^{1 / p}\left(\int_{x_{i}}^{x_{i+1}} v^{-p^{\prime} / p}(s)\left(x_{i+1}-s\right)^{\alpha p^{\prime}} d s\right)^{1 / p^{\prime}} \\
& \quad \leq C\left(\frac{b-x_{i}}{x_{i+1}-x_{i}}\right)^{\alpha}\left(\int_{x_{i}}^{x_{i+1}}|f|^{p}(s) v(s) d s\right)^{1 / p}\left(\int_{x_{i+1}}^{x_{i+2}} u(s) d s\right)^{-1 / p}\left(x_{i+2}-x_{i}\right)^{1+\alpha} \\
& \quad \leq C\left(b-x_{i}\right)^{\alpha}\left(x_{i+2}-x_{i}\right)\left(\frac{\int_{x_{i}}^{x}|f|^{p}(s) v(s) d s}{\int_{x_{i}}^{x} u(s) d s}\right)^{1 / p} \\
& \quad \leq C\left[M_{u}^{-}\left(|f|^{p} v u^{-1}\right)\right]^{1 / p}(x) \int_{x_{i}}^{x_{i+2}}(b-s)^{\alpha} d s .
\end{aligned}
$$

Now, summing up in $i$, we get

$$
\mathrm{I} \leq C\left[M_{u}^{-}\left(|f|^{p} v u^{-1}\right)\right]^{1 / p}(x) \int_{a}^{x_{N}}(b-s)^{\alpha} d s \leq C\left[M_{u}^{-}\left(|f|^{p} v u^{-1}\right)\right]^{1 / p}(x)(b-a)^{1+\alpha} \text {. }
$$

Finally, putting together the estimates of I and II, we are done.

Proof of THEOREM 2.2. The proof is similar to that of Theorem 2.1. We give just a sketch. First, (ii) follows from (i) on applying the standard argument to $\chi_{E \cap(a, b)}$. The converse follows from the fact that (ii) implies $N_{\alpha}^{-} \chi_{E}(x) \leq C\left[M_{u}^{-}\left(\chi_{E} v u^{-1}\right)\right]^{1 / p}(x)$, for some constant $C$ independent of the measurable subset $E$. To prove the above inequality, let $x \in \mathbb{R},(a, b) \in \mathscr{A}_{x}$ and assume first that $4 \int_{b}^{x} u>\int_{a}^{x} u$. Since $(u, v)$ 
satisfies $R A_{p, \alpha}^{-}$we obtain

$$
\begin{aligned}
\int_{a}^{b} \chi_{E}(s)(b-s)^{\alpha} d s & \leq C(x-a)^{1+\alpha}\left(\int_{a}^{b} \chi_{E}(s) v(s) d s\right)^{1 / p}\left(\int_{b}^{x} u(s) d s\right)^{-1 / p} \\
& \leq C(b-a)^{1+\alpha}\left(\int_{a}^{x} \chi_{E}(s) v(s) d s\right)^{1 / p}\left(\int_{a}^{x} u\right)^{-1 / p} \\
& \leq C(b-a)^{1+\alpha}\left[M_{u}^{-}\left(\chi_{E} v u^{-1}\right)\right]^{1 / p}(x)
\end{aligned}
$$

If $4 \int_{b}^{x} u \leq \int_{a}^{x} u$, we proceed as in the proof of Proposition 2.7.

\section{Singular integrals in the Cesàro sense}

Let $K$ be a Calderón-Zygmund kernel on $\mathbb{R}$, that is, a function $K \in L_{\text {loc }}^{1}(\mathbb{R} \backslash\{0\})$ such that

(1) $|K(x)| \leq C|x|^{-1},|x|>0$,

(2) $|K(x-y)-K(x)| \leq C|y||x|^{-2}$, if $|x|>2|y|>0$,

(3) $\left|\int_{\epsilon<|x|<N} K(x) d x\right| \leq C$ for all $\epsilon$ and all $N$ with $0<\epsilon<N$.

If the limit $\lim _{\epsilon \rightarrow 0^{+}} \int_{\epsilon<|y|<1} K(y) d y$ exists, then the principal-value singular integral

$$
T f(x)=\lim _{\epsilon \rightarrow 0^{+}} \int_{|x-y|>\epsilon} K(x-y) f(y) d y
$$

exists for $f \in L^{p}(w d x)$ with $w$ in the Muckenhoupt class $A_{p}$ (see for instance [4]). When the kernel $K$ has support in $(0, \infty)$ (or in $(-\infty, 0))$, then, as proved in [1], the same result holds for a wider class of weights, more precisely for weights in the Sawyer class $A_{p}^{-} \equiv A_{p, 0}^{-}$([7]).

Recently, in [2], we studied the existence in the Cesàro- $\alpha$ sense of the singular integral associated with $K$ for $-1<\alpha<0$, that is, the existence of the limit

$$
\lim _{\epsilon \rightarrow 0^{+}} T_{\epsilon, \alpha} f(x)=\lim _{\epsilon \rightarrow 0^{+}} \int_{|x-y|>\epsilon} f(y) K(x-y)\left(1-\frac{\epsilon}{|x-y|}\right)^{\alpha} d y
$$

in the setting of weighted $L^{p}$-spaces. The aim in this section is to obtain sharper results on singular integrals in the Cesàro- $\alpha$ sense for kernels with support in $(0, \infty)$ (or in $(-\infty, 0)$ ). We shall show, using the results of Section 2 , that, for these kernels, the results in [2] are true for a wider class of weights.

One of the key steps in [2] is the pointwise estimate from above of the maximal operator $T_{\alpha}^{*} f=\sup _{\epsilon>0}\left|T_{\epsilon, \alpha} f\right|$ by $C\left(M_{\alpha} f+T_{0}^{*} f\right)$. If the support of $K$ is contained in $(0, \infty)$, then we can improve this estimate by replacing $M_{\alpha}$ with a smaller operator $M_{\alpha}^{-}$. 
Proposition 3.1. Let $-1<\alpha<0$ and let $K$ be a Calderón-Zygmund kernel with support contained in $(0, \infty)$. If $f$ is a measurable function such that $T_{\epsilon, \alpha} f(x)$ is defined for every $\epsilon>0$, then there exists $C>0$ independent of $f$ such that

$$
T_{\alpha}^{*} f(x) \leq C\left[M_{\alpha}^{-} f(x)+T_{0}^{*} f(x)\right] .
$$

The proof is similar to that of [2, Proposition 2.5], and is therefore omitted. This proposition together with Theorem 2.1 in this paper and [1, Theorem 2.1] enables us to prove the following result.

THEOREM 3.2. Let $-1<\alpha<0$ and let $K$ be a Calderón-Zygmund kernel with support contained in $(0, \infty)$ such that the limit

$$
\lim _{\epsilon \rightarrow 0^{+}} \int_{\epsilon}^{1} K(y)\left(1-\frac{\epsilon}{y}\right)^{\alpha} d y
$$

exists. Then the singular integral exists a.e. in the Cesàro- $\alpha$ sense if $f \in L^{p}(w d x)$ with $p(1+\alpha)>1$ and $w \in A_{p, \alpha}^{-}$(the pair $(w, w)$ satisfies $\left.A_{p, \alpha}^{-}\right)$.

To prove the theorem we have to show first that the truncations $T_{\epsilon, \alpha} f$ are well defined for $f \in L^{p}(w d x), w \in A_{p, \alpha}^{-}, p(1+\alpha)>1$. This can be proved as in [2, Theorem 2.7]. The rest of the proof is a consequence of the following facts: (i) the existence of the $\operatorname{limit}_{\lim _{\epsilon \rightarrow 0^{+}}} T_{\epsilon, \alpha} f$ for $f$ in a dense class and (ii) the weak type $(p, p)$ boundedness with respect to $w(x) d x$ of the maximal operator $T_{\alpha}^{*}$. The former is clear since $L^{p}(w d x) \cap L^{p}(d x)$ is dense in $L^{p}(w d x)$ and the convergence holds for $f \in L^{p}(w d x) \cap L^{p}(d x)$ by [2, Theorem 2.7]. The latter immediately follows from Proposition 3.1, Theorem 2.1, [1, Theorem 2.1] and the easy implication $w \in A_{p, \alpha}^{-} \Rightarrow w \in A_{p, 0}^{-} \equiv A_{p}^{-}$.

REMARK. In particular, the result holds if $w$ belongs to the Sawyer's class [7] $A_{p(1+\alpha)}^{-}$ since $A_{p(1+\alpha)}^{-} \subset A_{p, \alpha}^{-}$. This inclusion follows from $A_{r}^{-} \subset A_{p, \alpha}^{-}, 1<r<p(1+\alpha)$, which is true by Hölder's inequality and the implication $w \in A_{p(1+\alpha)}^{-} \Rightarrow w \in A_{r}^{-}$for some $r<p(1+\alpha)$ (see [7] or [6]).

We do not know whether $A_{p(1+\alpha)}^{-}$is equal to $A_{p, \alpha}^{-}$for $\alpha<0$ and $p>1 /(1+\alpha)$ but in the endpoint $p=1 /(1+\alpha)$ it is possible to see that $R A_{1 /(1+\alpha), \alpha}^{-}$equals the Sawyer's class $A_{1}^{-}$. The proof of this fact is similar to the proof of [3, Proposition 6.5]. Then, following the steps in the proof of [2, Theorem 2.7] and using the corresponding results in this paper and in [1] we have our next result.

THEOREM 3.3. Let $\alpha$ and $K$ be as in Theorem 3.2. If $f$ belongs to the Lorentz space $L_{1 /(1+\alpha), 1}(\omega d x)=\left\{f: \int_{0}^{\infty}[\omega(\{x:|f(x)|>t\})]^{1+\alpha} d t<\infty\right\}$ and $\omega \in A_{1}^{-}$, then the singular integral exists a.e. in the Cesàro- $\alpha$ sense. 
EXAMPLE. Observe that the Calderón-Zygmund kernel

$$
K(x)=\frac{1}{x} \frac{\sin (\log x)}{\log x} \chi_{(0, \infty)}(x)
$$

given in [1] satisfies the condition in Theorem 3.2, that is,

$$
\lim _{\epsilon \rightarrow 0^{+}} \int_{\epsilon}^{1} K(y)\left(1-\frac{\epsilon}{y}\right)^{\alpha} d y
$$

exists. In fact, for any $0<\epsilon<1 / 2$, if $\Omega(x)=\sin x / x$, then

$$
\begin{gathered}
\int_{\epsilon}^{1} K(y)\left(1-\frac{\epsilon}{y}\right)^{\alpha} d y=\int_{\epsilon}^{1} \frac{\Omega(\log y)}{y}\left(1-\frac{\epsilon}{y}\right)^{\alpha} d y \\
=\int_{\epsilon}^{2 \epsilon} \cdots d y+\int_{2 \epsilon}^{1} \cdots d y=\mathrm{I}+\mathrm{II} .
\end{gathered}
$$

Applying the Hölder inequality to $I$ with $p>1 /(1+\alpha)$ and changing variables we obtain

$$
\begin{aligned}
|\mathrm{I}| & \leq\left(\int_{\epsilon}^{2 \epsilon} \frac{|\Omega(\log y)|^{p}}{y} d y\right)^{1 / p}\left(\int_{\epsilon}^{2 \epsilon}\left(1-\frac{\epsilon}{y}\right)^{\alpha p^{\prime}} \frac{1}{y} d y\right)^{1 / p^{\prime}} \\
& \leq C\left(\int_{\log \epsilon}^{\log 2 \epsilon}|\Omega(t)|^{p} d t\right)^{1 / p} \leq C\left(\int_{\log \epsilon}^{\log 2 \epsilon} \frac{1}{|t|^{p}} d t\right)^{1 / p}
\end{aligned}
$$

and therefore $\lim _{\epsilon \rightarrow 0^{+}} I=0$. On the other hand,

$$
\mathrm{II}=\int_{2 \epsilon}^{1} \frac{\Omega(\log y)}{y}\left[\left(1-\frac{\epsilon}{y}\right)^{\alpha}-1\right] d y+\int_{2 \epsilon}^{1} \frac{\Omega(\log y)}{y} d y=\mathrm{III}+\mathrm{IV} .
$$

Clearly, by changing the variables, we see that $\lim _{\epsilon \rightarrow 0^{+}}$IV exists. In order to estimate III, we apply the mean value theorem to get

$$
|\mathrm{III}| \leq|\alpha| \int_{2 \epsilon}^{1}|\Omega(\log y)|\left(1-\frac{\epsilon}{y}\right)^{\alpha-1} \frac{\epsilon}{y^{2}} d y .
$$

Changing variables again, we obtain

$$
|\mathrm{III}| \leq|\alpha| \int_{\epsilon}^{1 / 2}|\Omega(\log (\epsilon / t))|(1-t)^{\alpha-1} d t .
$$

Finally, $\lim _{\epsilon \rightarrow 0^{+}} \mathrm{III}=0$, applying the dominated convergence theorem and the facts that $\Omega$ is bounded and $\lim _{f \rightarrow 0^{+}} \Omega(\log (\epsilon / t))=0$. 
REMARK. If we do not assume anything about the support of $K$, then Theorem 3.2 is valid for weights $w$ in $A_{p, \alpha}$. The proof is similar to the proof of [2, Theorem 2.7] but using Theorem 2.3 instead of [2, Theorem 2.6].

An analogous comment can be written about Theorem 3.3, that is, Theorem 3.3 is valid for Calderón-Zygmund kernels and weights $w$ in the Muckenhoupt Class $A_{1}$ (in fact, notice that this result is contained in [2, Theorem 2.7]).

\section{References}

[1] H. Aimar, L. Forzani and F. J. Martín-Reyes, 'On weighted inequalities for singular integrals', Proc. Amer. Math. Soc. 125 (1997), 2057-2064.

[2] A. L. Bernardis and F. J. Martín-Reyes, 'Singular integrals in the Cesàro sense', J. Fourier Anal. 6 (2000), 143-152.

[3] _Weighted inequalities for a maximal function on the real line', Proc. Roy. Soc. Edinburgh Sect. A 131 (2001), 267-277.

[4] J. García-Cuerva and J. L. Rubio de Francia, Weighted norm inequalities and related topics, NorthHolland Math. Stud., 116; Notas de Matemática (Math. Notes), 104 (North-Holland, Amsterdam, 1985).

[5] W. Jourkat and J. Troutman, 'Maximal inequalities related to generalized a.e. continuity', Trans. Amer. Math. Soc. 252 (1979), 49-64.

[6] F. J. Martín-Reyes, 'New proofs of weighted inequalities for the one-sided Hardy-Littlewood maximal functions', Proc. Amer. Math. Soc. 117 (1993), 691-698.

[7] E. Sawyer, 'Weighted inequalities for the one-sided Hardy-Littlewood maximal functions', Trans. Amer. Math. Soc. 297 (1986), 53-61.

IMAL

Güemes 3450

(3000) Santa Fe

Argentina

e-mail: bernard@ceride.gov.ar
Departamento de Análisis Matemático

Facultad de Ciencias

Universidad de Málaga

29071 Málaga

Spain

e-mail: martin@anamat.cie.uma.es 